\title{
Bioherbicidal potential of some allelopathic agroforestry and fruit plant species against Lepidium sativum
}

\author{
Shagufta Perveen ${ }^{1}$, Muhammad Yousaf ${ }^{1}$, Muhammad Naeem Mushtaq ${ }^{2 *}$, Nighat Sarwar ${ }^{3}$, \\ Muhammad Yahya Khan ${ }^{2}$ and Sajid Mahmood Nadeem ${ }^{2}$ \\ ${ }^{1}$ Department of Chemistry, Government College University, Faisalabad, Pakistan \\ ${ }^{2}$ University of Agriculture Faisalabad, Sub Campus Burewala, Pakistan \\ ${ }^{3}$ Plant Protection Division, Nuclear Institute for Agriculture and Biology, Faisalabad, Pakistan
}

\begin{abstract}
Herbicide resistance and environmental pollution are the risks associated with chemical control of weeds. Allelopathic plant extracts may be exploited for weed management as an alternative to commercial herbicides. Before development of allelochemical-based eco-friendly herbicides, bioherbicidal potential of plants need to be evaluated. Present study was conducted to evaluate phytotoxic allelopathic impact of leaf extract from eight agroforestry and fruit plant species against Lepidium sativum. The results showed that all plant species delayed germination and inhibited root length, shoot length and seedling dry weight of Lepidium sativum. Four plant species such as Moringa oleifera, Mangifera indica, Albizia procera and Delonix regia were most phytotoxic with Lepidium sativum root growth inhibition of $\geq 85 \%$ as compared with control and seedling persistence index $<30 \%$ of control. Phenolic contents were maximum in Mangifera indica (137 $\mathrm{mg} \mathrm{g}^{-1}$ leaf dry weight) followed by Delonix regia (130 $m g g^{-1}$ leaf dry weight). The results suggest that phytotoxic action of leaf extract of plant species may be due to presence of phenolic allelochemicals that may be exploited further either directly for weed management or development of bioherbicides.
\end{abstract}

Keywords: Allelopathy, allelochemicals, growth inhibition, phytotoxicity, weed management

\section{Introduction}

Weeds have been a major threat to crop productivity in agroecosystems since start of the agriculture on earth and lack of weed control is a pressing concern expressed by the farmers (Stokstad, 2013). Herbicide use have been the most effective way to control weeds especially in the last few decades. The share of herbicides in total pesticide use in the world is about $47.5 \%$ with an annual consumption of 0.95 million tonnes (De et al., 2014). Nonetheless, there is growing public, scientific and farmers' concern regarding harmful impact of herbicides on environment and development of herbicide resistant weeds (Edwards, 2013, Dayan and Duke, 2014). Presently, 255 weed species (148 dicots and 107 monocots) have developed resistance to 163 herbicides in 92 crops in 70 countries (Heap, 2018). Furthermore, in the last 20 years, no new mode of action of the herbicides has been reported (Dayan and Duke, 2014; Romano-Armada et al., 2017). Keeping in view all abovementioned problems associated with use of commercial herbicides, there is growing need for alternative options for weed control in agroecosystems.

Higher plants produce various secondary metabolites (natural products) which may be released into the environment as allelochemicals to inhibit growth of the other plant species including weeds (Cheng and Cheng, 2015). Phenolic compounds are a major group of allelochemicals ranging from phenols, hydroxy acids, aldehydes, benzoic acids cinnamic acids, coumarins, tannins and flavonoids. They are produced by various plant species and their inhibitory effect on crops and weeds have been well reported (John, 2012, Mushtaq et al., 2013; Tariq et al., 2018; Perveen et al., 2019). At times, the secondary metabolites are not released by plants into the environment; however, they still act as natural phytotoxins. Either exploited as allelochemicals or phytotoxins, the natural products offer a viable option for weed control in agroecosystems as an alternative to commercial herbicides.

Many agroforestry and fruit plant species have been reported as allelopathic thus showing bioherbicidal potential to be used for management of weeds. For example, Dalbergia sissoo leaf extract inhibited germination and growth of maize, pearl millet and rice (Akhtar et al., 2010). Mulberry leaf extract suppressed the growth of bermuda grass (Haq et al., 2010). Growth of understory plant species was reduced by allelochemicals from Delonix regia (Chou and Leu, 1992).

\footnotetext{
*Email: mnmushtaq@gmail.com
} 
Though few reports are available regarding phytotoxic potential of common agroforestry and fruit plant species (Moringa oleifera, Morus alba, Mangifera indica, Albizia lebbeck, Albizia procera, Delonix regia, Ziziphus jujube, Ziziphus mauritiana) of Asia including Pakistan; nevertheless, these plants have been tested against different target crops and weed species with extreme variation in the extract concentrations from species to species under variable test conditions, which makes it difficult to decide the most potent allelopathic plant species to exploit further for bioherbicidal efficacy (Chou and Leu, 1992, Uddin et al., 2007; Alshahrani et al., 2009; Haq et al., 2010; Phiri and Mbewe, 2010; Piyatida and Kato-Noguchi, 2010; Saleem et al., 2013). The present study was therefore conducted to explore and compare bioherbicidal potential of plant species using an established model test plant species (Lepidium sativum) for allelopathic studies under identical controlled environment for germination, early seedling growth and total phenolic contents of all donor plant species. Lepidium sativum is a leafy edible plant which has been used as a test species for initial screening in laboratory bioassays to evaluate the phytotoxic effect of allelochemicals and/or natural phytotoxins (Vaughn and Berhow, 1999; Macias et al., 2000; Perveen et al., 2015). Furthermore, we used modified equation to determine seedling persistence index, which has been seldom employed to evaluate persistence of target plant seedlings, for comparison of phytotoxic potential of multiple plant species and concentrations.

\section{Materials and Methods}

The extract was prepared as previously described (Perveen et al., 2015). Briefly, the leaves of tree species were collected from University of Agriculture, Faisalabad. The oven-dried leaves were ground to powder. The possible phytotoxins from leaf powder of each plant were extracted by adding $10 \mathrm{~g}$ powder to $100 \mathrm{~mL}$ distilled water on an ultrasonic device for 30 min. The extract was sieved through cheesecloth to obtain $10 \%$ extract. Further dilutions $(2.5,5$ and $7.5 \%)$ were made using distilled water.

The seeds of Lepidium sativum were surface sterilized with $2 \% \mathrm{NaClO}$ for 2 minutes and were rinsed with distilled water to remove traces of $\mathrm{NaClO}$. Ten seeds of Lepidium sativum were placed on a filter paper soaked with $4 \mathrm{~mL}$ of respective concertation of each species in Petri dishes $(9.5 \mathrm{~cm}$ diameter). The control ( $0 \%$ extract) received only distilled water. The petri dishes were sealed and placed in a growth cabinet at $24 \pm 2^{\circ} \mathrm{C}$ and $14 / 10$ (light/dark) for 6 days. The germinated seeds were counted daily and time to start germination was noted. Mean germination time (MGT) was calculated by following formula (Ellis and Roberts, 1981):

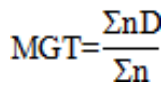

where $n=$ number of newly germinated seeds at time $D ; D=$ Number of days from the sowing, $\sum \mathrm{n}=$ Final germination

Germination index (GI) was calculated by following formula as reviewed and described earlier (Ranal and Santana, 2006):

$\mathrm{GI}=\frac{\text { Number of germinated seeds }}{\text { Days of first count }}+\ldots+\frac{\text { Number of germinated seeds }}{\text { Days of final count }}$

Root and shoot length and dry weight of seedlings were measured 6 days after sowing. Seedling persistence index (SPI) was calculated using formula modified from the previously reported (Mishra and Misra, 1997) and expressed as $\%$ of control:

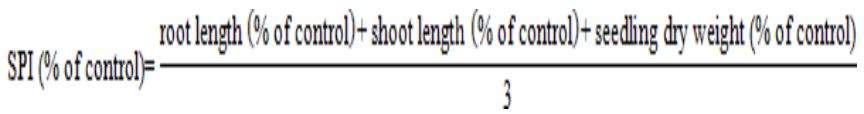

Total phenolic compounds from leaf extract of eight allelopathic plant species were measured using FolinCiocalteu reagent (Saleem et al., 2013). Chlorogenic acid was used as a standard and its concentrations (0-1000 ppm) were measured in the same way as leaf extract samples to draw a calibration curve and calculate phenolic contents. To $200 \mu \mathrm{L}$ of the samples or respective chlorogenic acid concentration or blank, $4 \mathrm{~mL}$ of $4 \% \mathrm{Na}_{2} \mathrm{CO}_{3}$ and $200 \mu \mathrm{L}$ of Folin-Ciocalteau reagent was added while vortex-mixing. Thereafter, the mixture was incubated at room temperature for $30 \mathrm{~min}$ and absorbance was measured at $750 \mathrm{~nm}$ on a spectrophotometer (Hitachi U-2800, Japan).

The results are reported as mean \pm SEM of five replications. The treatment means were statistically compared by ANOVA followed by post-hoc Duncan's Multiple Range Test (DMRT) at $p<5 \%$.

\section{Results}

\section{Effect of allelopathic plant leaf extract on germination of Lepidium sativum}

Leaf extract of all plant species significantly delayed time to start germination and mean germination time and reduced germination index in a concentration-dependent manner (Table 1). The time to start germination and mean germination time was delayed by $\geq 1$ day as compared with control after application of $10 \%$ leaf extract of all donor plant species except Morus alba. The same concentration $(10 \%)$ of Moringa oleifera, Mangifera indica, Albizia procera and Delonix regia decreased germination index by $\geq 50$. The final germination was significantly inhibited by plant species at any concentration except Moringa oleifera that reduced final germination by $26 \%$ and Mangifera indica where no seed was germinated at $10 \%$ leaf extract (Table 1). 
Table 1: Inhibitory effect of allelopathic aqueous leaf extract of agroforestry and fruit tree species on germination of Lepidium sativum

\begin{tabular}{|c|c|c|c|c|c|}
\hline $\begin{array}{c}\text { Name of plant } \\
\text { species }\end{array}$ & $\begin{array}{c}\text { Extract } \\
\text { concentration } \\
(\%)\end{array}$ & $\begin{array}{l}\text { Time to start } \\
\text { germination } \\
\text { (days) }\end{array}$ & $\begin{array}{c}\text { Mean } \\
\text { germination } \\
\text { time (days) }\end{array}$ & $\begin{array}{l}\text { Germination } \\
\text { index }\end{array}$ & $\begin{array}{c}\text { Final } \\
\text { germination } \\
(\%)\end{array}$ \\
\hline Control & 0.0 & $1.0 \pm 0.0 \mathrm{~g}$ & $1.0 \pm 0.0 q$ & $10 \pm 0.0 b c$ & $100 \pm 0.0 \mathrm{a}$ \\
\hline \multirow[t]{4}{*}{$\begin{array}{c}\text { Moringa } \\
\text { oleifera }\end{array}$} & 2.5 & $\begin{array}{l}2.0 \pm 0.0 \mathrm{f} \\
\quad(100)\end{array}$ & $\begin{array}{c}2.0 \pm 0.01 \mathrm{mno} \\
(100)\end{array}$ & $\begin{array}{c}5.10 \pm 0.09 \text { efghijk } \\
(-49)\end{array}$ & $\begin{array}{c}100 \pm 0.0 \mathrm{a} \\
(0)\end{array}$ \\
\hline & 5.0 & $\begin{array}{l}2.0 \pm 0.0 \mathrm{f} \\
\quad(100)\end{array}$ & $\begin{array}{c}2.46 \pm 0.21 \mathrm{ghi} \\
(146)\end{array}$ & $\begin{array}{c}6.22 \pm 0.45 \mathrm{defg} \\
(-37.8)\end{array}$ & $\begin{array}{c}98 \pm 1.88 a \\
(-2)\end{array}$ \\
\hline & 7.5 & $\begin{array}{l}2.0 \pm 0.0 \mathrm{f} \\
(100)\end{array}$ & $\begin{array}{c}2.24 \pm 0.14 \mathrm{ijkl} \\
(124)\end{array}$ & $\begin{array}{c}5.53 \pm 0.45 \text { fghij } \\
(-44.7)\end{array}$ & $\begin{array}{c}100 \pm 0.0 \mathrm{a} \\
(0)\end{array}$ \\
\hline & 10 & $\begin{array}{c}3.0 \pm 0.0 b c \\
(200)\end{array}$ & $\begin{array}{c}3.0 \pm 0.0 \mathrm{~d} \\
(200)\end{array}$ & $\begin{array}{c}2.42 \pm 0.07 \mathrm{no} \\
(-75.8)\end{array}$ & $\begin{array}{c}74 \pm 2.31 c \\
(-26)\end{array}$ \\
\hline \multirow[t]{4}{*}{ Morus alba } & 2.5 & $\begin{array}{c}1.0 \pm 0.0 \mathrm{~g} \\
(0)\end{array}$ & $\begin{array}{c}1.13 \pm 0.12 q \\
(13)\end{array}$ & $\begin{array}{c}10.0 \pm 0.0 \mathrm{bc} \\
(0)\end{array}$ & $\begin{array}{c}100 \pm 0.0 \mathrm{a} \\
(0)\end{array}$ \\
\hline & 5.0 & $\begin{array}{c}1.0 \pm 0.0 \mathrm{~g} \\
(0)\end{array}$ & $\begin{array}{c}1.70 \pm 0.11 \mathrm{op} \\
(70)\end{array}$ & $\begin{array}{c}12.40 \pm 0.98 \mathrm{a} \\
(24)\end{array}$ & $\begin{array}{c}100 \pm 0.0 \mathrm{a} \\
(0)\end{array}$ \\
\hline & 7.5 & $\begin{array}{c}1.2 \pm 0.19 \mathrm{~g} \\
(20)\end{array}$ & $\begin{array}{c}1.97 \pm 0.131 \mathrm{mno} \\
(97)\end{array}$ & $\begin{array}{c}9.53 \pm 1.17 b c \\
(-4.7)\end{array}$ & $\begin{array}{c}100 \pm 0.0 \mathrm{a} \\
(0)\end{array}$ \\
\hline & 10 & $\begin{array}{c}1.8 \pm 0.19 \mathrm{f} \\
(80)\end{array}$ & $\begin{array}{c}1.94 \pm 0.051 \mathrm{mno} \\
(94)\end{array}$ & $\begin{array}{c}5.80 \pm 0.80 \text { defgh } \\
(-42)\end{array}$ & $\begin{array}{c}100 \pm 0.0 \mathrm{a} \\
(0)\end{array}$ \\
\hline \multirow[t]{4}{*}{$\begin{array}{c}\text { Mangifera } \\
\text { indica }\end{array}$} & 2.5 & $\begin{array}{c}1.0 \pm 0.0 \mathrm{~g} \\
(0)\end{array}$ & $\begin{array}{c}4.0 \pm 017 c \\
(300)\end{array}$ & $\begin{array}{c}10.5 \pm 0.98 b c \\
(5)\end{array}$ & $\begin{array}{c}96 \pm 3.76 a \\
(-4)\end{array}$ \\
\hline & 5.0 & $\begin{array}{c}3.2 \pm 0.35 b \\
(220)\end{array}$ & $\begin{array}{c}5.58 \pm 0.14 a \\
(458)\end{array}$ & $\begin{array}{c}5.58 \pm 0.21 \text { defghi } \\
(-44.2)\end{array}$ & $\begin{array}{c}98 \pm 1.88 \mathrm{a} \\
(-2)\end{array}$ \\
\hline & 7.5 & $\begin{array}{c}3.80 \pm 0.20 \mathrm{a} \\
(280)\end{array}$ & $\begin{array}{c}5.22 \pm 0.06 b \\
(422)\end{array}$ & $\begin{array}{c}3.21 \pm 0.311 \mathrm{mn} \\
(-67.9)\end{array}$ & $\begin{array}{c}74 \pm 5.64 c \\
(-26)\end{array}$ \\
\hline & 10 & - & - & - & - \\
\hline \multirow[t]{4}{*}{ Albizia lebbeck } & 2.5 & $\begin{array}{l}2.0 \pm 0.0 \mathrm{f} \\
(100)\end{array}$ & $\begin{array}{c}1.90 \pm 0.09 \text { mnop } \\
(90)\end{array}$ & $\begin{array}{c}10.8 \pm 0.80 b \\
(-67.9)\end{array}$ & $\begin{array}{c}100 \pm 0.0 \mathrm{a} \\
(0)\end{array}$ \\
\hline & 5.0 & $\begin{array}{c}1.0 \pm 0.0 \mathrm{~g} \\
\quad(0)\end{array}$ & $\begin{array}{c}1.87 \pm 0.02 \text { nop } \\
(87)\end{array}$ & $\begin{array}{c}6.40 \pm 0.24 \mathrm{de} \\
(-36)\end{array}$ & $\begin{array}{c}100 \pm 0.0 \mathrm{a} \\
(0)\end{array}$ \\
\hline & 7.5 & $\begin{array}{l}2.0 \pm 0.0 \mathrm{f} \\
\quad(100)\end{array}$ & $\begin{array}{c}2.0 \pm 0.01 \mathrm{mno} \\
(100)\end{array}$ & $\begin{array}{c}5.0 \pm 0.0 \mathrm{ghijk} \\
(-50)\end{array}$ & $\begin{array}{c}100 \pm 0.0 \mathrm{a} \\
(0)\end{array}$ \\
\hline & 10 & $\begin{array}{c}2.0 \pm 0.0 \mathrm{f} \\
(100)\end{array}$ & $\begin{array}{c}2.20 \pm 0.12 \mathrm{ijklm} \\
(-220)\end{array}$ & $\begin{array}{c}6.13 \pm 0.69 \mathrm{defg} \\
(-38.7)\end{array}$ & $\begin{array}{c}100 \pm 0.0 \mathrm{a} \\
(0)\end{array}$ \\
\hline \multirow[t]{4}{*}{ Albizia procera } & 2.5 & $\begin{array}{c}1.0 \pm 0.0 \mathrm{~g} \\
(0)\end{array}$ & $\begin{array}{c}2.0 \pm 0.01 \mathrm{mno} \\
(100)\end{array}$ & $\begin{array}{c}5.0 \pm 0.0 \text { ghijk } \\
(-50)\end{array}$ & $\begin{array}{c}100 \pm 0.0 \mathrm{a} \\
(0)\end{array}$ \\
\hline & 5.0 & $\begin{array}{c}2.4 \pm 0.20 \mathrm{e} \\
(140)\end{array}$ & $\begin{array}{c}2.40 \pm 0.23 \mathrm{hij} \\
(-240)\end{array}$ & $\begin{array}{c}4.33 \pm 0.4 \mathrm{ijkl} \\
(-56.7)\end{array}$ & $\begin{array}{c}100 \pm 0.0 \mathrm{a} \\
(0)\end{array}$ \\
\hline & 7.5 & $\begin{array}{c}2.60 \pm 0.24 \mathrm{de} \\
(160)\end{array}$ & $\begin{array}{c}2.60 \pm 0.23 \mathrm{fgh} \\
(-240)\end{array}$ & $\begin{array}{c}3.0 \pm 0.41 \mathrm{jkl} \\
(-70)\end{array}$ & $\begin{array}{c}100 \pm 0.0 \mathrm{a} \\
(0)\end{array}$ \\
\hline & 10 & $\begin{array}{c}2.0 \pm 0.0 \mathrm{f} \\
(100)\end{array}$ & $\begin{array}{c}2.0 \pm 0.01 \mathrm{mno} \\
(100)\end{array}$ & $\begin{array}{c}5.0 \pm 0.0 \mathrm{ghijk} \\
(-50)\end{array}$ & $\begin{array}{c}100 \pm 0.0 \mathrm{a} \\
(0)\end{array}$ \\
\hline \multirow[t]{4}{*}{ Delonix regia } & 2.5 & $\begin{array}{c}1.0 \pm 0.0 \mathrm{~g} \\
(0)\end{array}$ & $\begin{array}{c}1.0 \pm 0.0 \mathrm{q} \\
(0)\end{array}$ & $\begin{array}{c}10.0 \pm 0.0 \mathrm{bc} \\
(0)\end{array}$ & $\begin{array}{c}100 \pm 0.0 \mathrm{a} \\
(0)\end{array}$ \\
\hline & 5.0 & $\begin{array}{l}2.0 \pm 0.0 \mathrm{f} \\
\quad(100)\end{array}$ & $\begin{array}{c}2.0 \pm 0.01 \mathrm{mno} \\
(100)\end{array}$ & $\begin{array}{c}5.20 \pm 0.12 \text { efghij } \\
(-48)\end{array}$ & $\begin{array}{c}100 \pm 0.0 \mathrm{a} \\
(0)\end{array}$ \\
\hline & 7.5 & $\begin{array}{l}2.0 \pm 0.0 \mathrm{f} \\
(100)\end{array}$ & $\begin{array}{c}2.0 \pm 0.01 \mathrm{mnop} \\
(100)\end{array}$ & $\begin{array}{c}5.0 \pm 0.0 \mathrm{ghijk} \\
(-50)\end{array}$ & $\begin{array}{c}100 \pm 0.0 \mathrm{a} \\
(0)\end{array}$ \\
\hline & 10 & $\begin{array}{c}2.0 \pm 0.0 \mathrm{f} \\
(100)\end{array}$ & $\begin{array}{c}2.0 \pm 0.0 \mathrm{klmno} \\
(100)\end{array}$ & $\begin{array}{c}5.0 \pm 0.0 \text { fghijk } \\
(-50)\end{array}$ & $\begin{array}{c}100 \pm 0.0 \mathrm{a} \\
(0)\end{array}$ \\
\hline
\end{tabular}




\begin{tabular}{llllll}
\hline Ziziphus jujube & 2.5 & $\begin{array}{l}1.0 \pm 0.0 \mathrm{~g} \\
(0)\end{array}$ & $\begin{array}{l}1.69 \pm 0.01 \mathrm{op} \\
(-240)\end{array}$ & $\begin{array}{l}9.4 \pm 0.0 \mathrm{c} \\
(-6)\end{array}$ & $\begin{array}{l}100 \pm 0.0 \mathrm{a} \\
(0)\end{array}$ \\
& 5.0 & $(100)$ & $(100)$ & $\begin{array}{l}0.0 \pm 0.12 \mathrm{ghijk} \\
(-50)\end{array}$ & $\begin{array}{l}100 \pm 0.0 \mathrm{a} \\
(0)\end{array}$ \\
& $2.0 \pm 0.0 \mathrm{f}$ & $2.32 \pm 0.12 \mathrm{hijk}$ & $6.60 \pm 0.0 \mathrm{~d}$ & $100 \pm 0.0 \mathrm{a}$ \\
& $(100)$ & $(-240)$ & $(-34)$ & $(0)$ \\
& 7.5 & $2.0 \pm 0.0 \mathrm{f}$ & $2.32 \pm 0.20 \mathrm{ijk}$ & $6.39 \pm 0.0 \mathrm{def}$ & $100 \pm 0.0 \mathrm{a}$ \\
& $(100)$ & $(-240)$ & $(-36.1)$ & $(0)$ \\
\hline Ziziphus & $2.0 \pm 0.0 \mathrm{f}$ & $1.64 \pm 0.02 \mathrm{p}$ & $10.6 \pm 0.24 \mathrm{bc}$ & $100 \pm 0.0 \mathrm{a}$ \\
mauritiana & 2.5 & $(100)$ & $(-240)$ & $(6)$ & $(0)$ \\
& $2.0 \pm 0.0 \mathrm{f}$ & $2.10 \pm 0.09 \mathrm{jklmn}$ & $6.0 \pm 1.0 \mathrm{defgh}$ & $100 \pm 0.0 \mathrm{a}$ \\
& 5.0 & $(100)$ & $(-240)$ & $(-40)$ & $(0)$ \\
& & $2.0 \pm 0.0 \mathrm{f}$ & $2.0 \pm 0.0 \mathrm{klmno}$ & $5.0 \pm 0.0 \mathrm{fghijk}$ & $34 \pm 0.0 \mathrm{e}$ \\
& 7.5 & $(100)$ & $(100)$ & $(-50)$ & $(-66)$ \\
& 10 & $2.0 \pm 0.0 \mathrm{f}$ & $2.74 \pm 0.13 \mathrm{defg}$ & $9.64 \pm 0.22 \mathrm{bc}$ & $100 \pm 0.0 \mathrm{a}$ \\
& $(100)$ & $(-240)$ & $(-3.6)$ & $(0)$ \\
\hline
\end{tabular}

Data are presented as mean \pm SEM; mean values having different letters in a column are statistically different at $p<0.05$; Values in parenthesis show \% (+) increase or (-) decrease as compared with control.

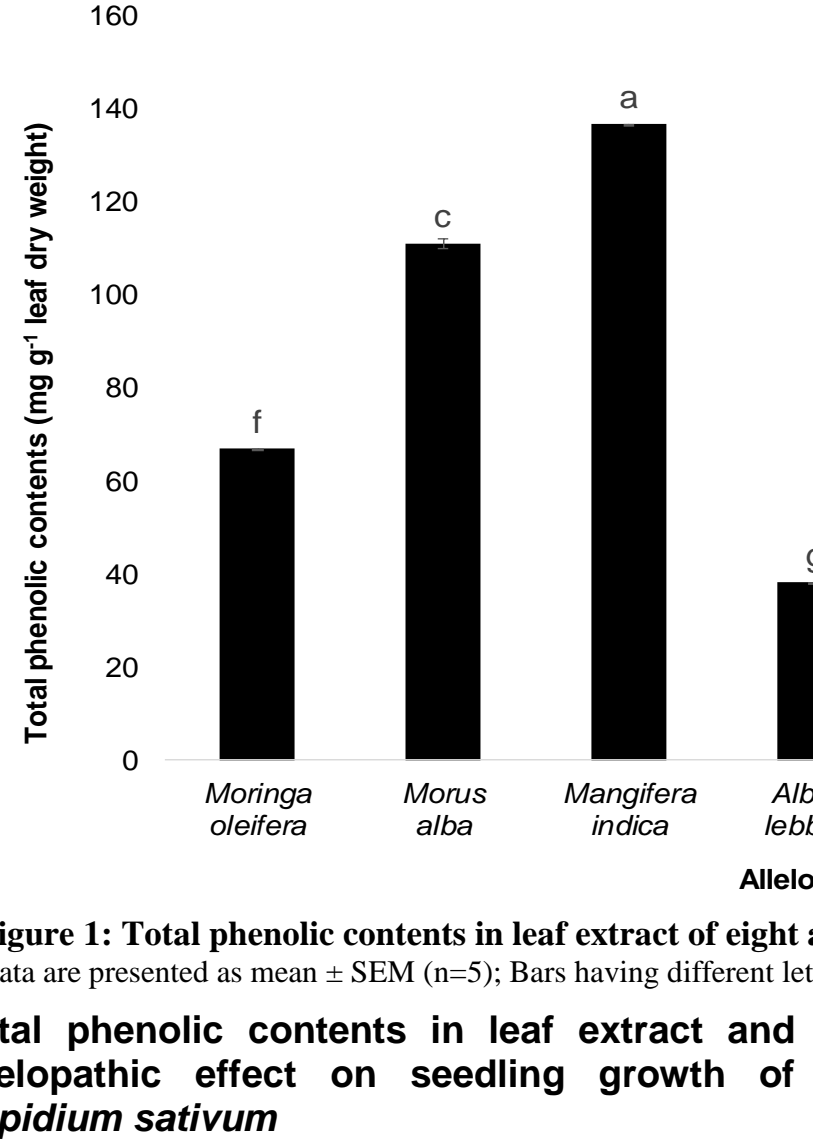

All plant species significantly suppressed shoot length, root length and seedling dry weight of Lepidium sativum at $\geq 5 \%$ leaf extract concertation (Table 2). Moringa oleifera, Mangifera indica, Albizia procera, Delonix regia and Ziziphus mauritiana inhibited root length by $\geq 85 \%$ and
Total phenolic contents in leaf extract and allelopathic effect on seedling growth of

\section{Lepidium sativum}
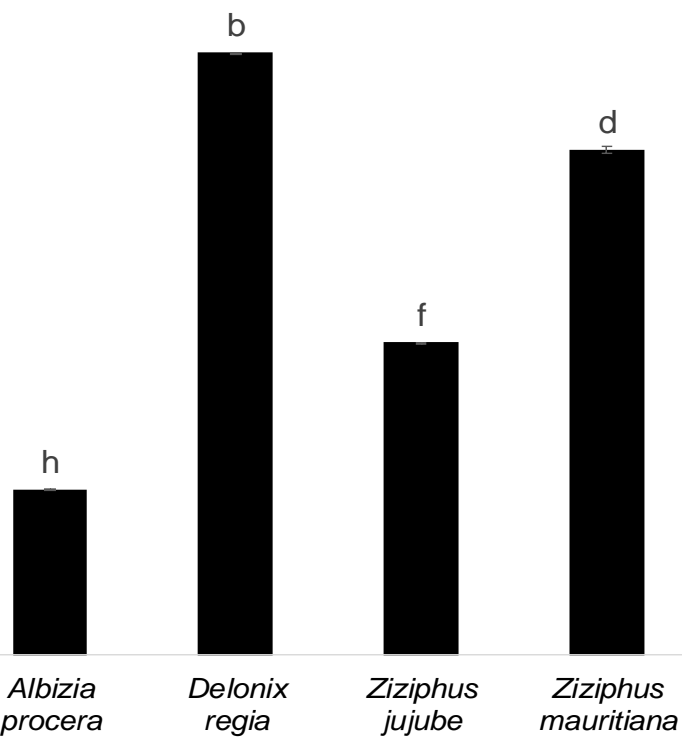
regia mauritiana

Allelopathic plant speceis

Figure 1: Total phenolic contents in leaf extract of eight allelopathic plant species.

seedling dry weight by $>55 \%$ at $10 \%$ leaf extract as compared with control (Table 2). Regarding seedling persistence index, four plant species such as Moringa oleifera, Mangifera indica, Albizia procera and Delonix regia were most phytotoxic with Lepidium sativum seedling persistence index $<30 \%$ of control (Table 2). Among test species, Mangifera indica had maximum (137 $\mathrm{mg} \mathrm{g}^{-1}$ leaf dry weight) phenolic contents followed by Delonix regia with $130 \mathrm{mg} \mathrm{g}^{-1}$ leaf dry weight (Figure 1). 
Table 2: Phytotoxic allelopathic effect of aqueous leaf extract of agroforestry and fruit tree species on seedling growth of Lepidium sativum

\begin{tabular}{|c|c|c|c|c|c|}
\hline $\begin{array}{l}\text { Name of } \\
\text { plant species }\end{array}$ & $\begin{array}{l}\text { Extract } \\
\text { concentration } \\
(\%)\end{array}$ & $\begin{array}{l}\text { Shoot length } \\
(\mathrm{cm})\end{array}$ & $\begin{array}{l}\text { Root length } \\
(\mathrm{cm})\end{array}$ & $\begin{array}{l}\text { Seedling dry } \\
\text { weight } \\
\text { (mg) } \\
\end{array}$ & $\begin{array}{l}\text { Seedling persistence } \\
\text { index (\% of control) }\end{array}$ \\
\hline \multirow{5}{*}{$\begin{array}{l}\text { Control } \\
\text { Moringa } \\
\text { oleifera }\end{array}$} & 0.0 & $4.16 \pm 0.04 d$ & $6.53 \pm 0.11 b$ & $10.60 \pm 0.38 a$ & 100 \\
\hline & 2.5 & $\begin{array}{l}5.57 \pm 0.13 a \\
(33.89)\end{array}$ & $\begin{array}{l}5.54 \pm 0.24 f \\
(-15.16)\end{array}$ & $\begin{array}{l}8.20 \pm 0.19 \mathrm{~cd} \\
(-22.64)\end{array}$ & 99 \\
\hline & 5.0 & $\begin{array}{l}4.56 \pm 0.07 b \\
(9.6)\end{array}$ & $\begin{array}{l}4.12 \pm 0.03 \mathrm{kl} \\
(-36.90)\end{array}$ & $\begin{array}{l}7.40 \pm 0.23 \mathrm{defg} \\
(-30.18)\end{array}$ & 81 \\
\hline & 7.5 & $\begin{array}{l}4.40 \pm 0.09 c \\
(5.7)\end{array}$ & $\begin{array}{l}2.12 \pm 0.02 \mathrm{~s} \\
(-67.53)\end{array}$ & $\begin{array}{l}4.60 \pm 0.23 \text { op } \\
(-56.60)\end{array}$ & 61 \\
\hline & 10 & $\begin{array}{l}2.17 \pm 0.01 \mathrm{kl} \\
(-47.3)\end{array}$ & $\begin{array}{l}0.91 \pm 0.05 \mathrm{vw} \\
(-86.06)\end{array}$ & $\begin{array}{l}2.80 \pm 0.19 s \\
(-73.58)\end{array}$ & 31 \\
\hline \multirow[t]{4}{*}{ Morus alba } & 2.5 & $\begin{array}{l}4.10 \pm 0.03 d \\
(-1.44)\end{array}$ & $\begin{array}{l}5.09 \pm 0.0 \mathrm{~h} \\
(-22.05)\end{array}$ & $\begin{array}{l}7.6 \pm 0.23 \mathrm{def} \\
(-28.30)\end{array}$ & 83 \\
\hline & 5.0 & $\begin{array}{l}3.20 \pm 0.02 g \\
(-23)\end{array}$ & $\begin{array}{l}4.06 \pm 0.02 \mathrm{i} \\
(-37.82)\end{array}$ & $\begin{array}{l}7.0 \pm 0.0 \text { efgh } \\
(-33.96)\end{array}$ & 68 \\
\hline & 7.5 & $\begin{array}{l}2.20 \pm 0.03 \mathrm{jk} \\
(-47)\end{array}$ & $\begin{array}{l}4.30 \pm 0.02 \mathrm{jk} \\
(-34)\end{array}$ & $\begin{array}{l}5.80 \pm 0.0 \mathrm{jkl} \\
(-45.28)\end{array}$ & 58 \\
\hline & 10 & $\begin{array}{l}2.06 \pm 0.02 \mathrm{kl} \\
(-50.5)\end{array}$ & $\begin{array}{l}3.75 \pm 0.05 \mathrm{~m} \\
(-42.57)\end{array}$ & $\begin{array}{l}4.80 \pm 0.19 \text { no } \\
(-54.71)\end{array}$ & 51 \\
\hline \multirow[t]{4}{*}{$\begin{array}{l}\text { Mangifera } \\
\text { indica }\end{array}$} & 2.5 & $\begin{array}{l}1.99 \pm 0.071 \\
(-52)\end{array}$ & $\begin{array}{l}4.19 \pm 0.03 \mathrm{kl} \\
(-35.83)\end{array}$ & $\begin{array}{l}7.40 \pm 0.23 \text { defg } \\
(-30.18)\end{array}$ & 61 \\
\hline & 5.0 & $\begin{array}{l}1.43 \pm 0.02 \mathrm{no} \\
(-85.7)\end{array}$ & $\begin{array}{l}1.44 \pm 0.02 u \\
(-77.94)\end{array}$ & $\begin{array}{l}6.40 \pm 0.23 \mathrm{hij} \\
(-39.62)\end{array}$ & 39 \\
\hline & 7.5 & $\begin{array}{l}0.52 \pm 0.04 r \\
(-65.6)\end{array}$ & $\begin{array}{l}0.36 \pm 0.03 x y \\
(-94.48)\end{array}$ & $\begin{array}{l}3.20 \pm 0.19 \mathrm{rs} \\
(-69.81)\end{array}$ & 16 \\
\hline & 10 & - & - & - & 0 \\
\hline \multirow[t]{4}{*}{$\begin{array}{l}\text { Albizia } \\
\text { lebbeck }\end{array}$} & 2.5 & $\begin{array}{l}4.58 \pm 0.04 b \\
(10.09)\end{array}$ & $\begin{array}{l}6.34 \pm 0.02 b c \\
(-2.90)\end{array}$ & $\begin{array}{l}9.0 \pm 0.0 \mathrm{~b} \\
(-15.09)\end{array}$ & 97 \\
\hline & 5.0 & $\begin{array}{l}3.77 \pm 0.05 \mathrm{e} \\
(-9.3)\end{array}$ & $\begin{array}{l}4.13 \pm 0.02 \mathrm{kl} \\
(-36.75)\end{array}$ & $\begin{array}{l}7.80 \pm 0.19 \mathrm{de} \\
(-26.41)\end{array}$ & 76 \\
\hline & 7.5 & $\begin{array}{l}3.42 \pm 0.04 f \\
(-17.8)\end{array}$ & $\begin{array}{l}4.18 \pm 0.02 \mathrm{kl} \\
(-35.98)\end{array}$ & $\begin{array}{l}7.20 \pm 0.19 \text { efgh } \\
(-32.07)\end{array}$ & 71 \\
\hline & 10 & $\begin{array}{l}2.16 \pm 0.02 \mathrm{jk} \\
(-48)\end{array}$ & $\begin{array}{l}2.08 \pm 0.02 \mathrm{~s} \\
(-68.14)\end{array}$ & $\begin{array}{l}5.80 \pm 0.19 \mathrm{jkl} \\
(-45.28)\end{array}$ & 46 \\
\hline \multirow[t]{4}{*}{$\begin{array}{l}\text { Albizia } \\
\text { procera }\end{array}$} & 2.5 & $\begin{array}{l}2.52 \pm 0.05 \mathrm{i} \\
(-39.42)\end{array}$ & $\begin{array}{l}2.36 \pm 0.01 r \\
(-63.85)\end{array}$ & $\begin{array}{l}7.0 \pm 0.0 \text { efgh } \\
(-33.96)\end{array}$ & 54 \\
\hline & 5.0 & $\begin{array}{l}1.84 \pm 0.03 m \\
(-55.7)\end{array}$ & $\begin{array}{l}1.11 \pm 0.01 \mathrm{v} \\
(-83.00)\end{array}$ & $\begin{array}{l}5.80 \pm 0.19 \mathrm{jkl} \\
(-45.28)\end{array}$ & 39 \\
\hline & 7.5 & $\begin{array}{l}1.48 \pm 0.04 \text { no } \\
(-64)\end{array}$ & $\begin{array}{l}1.12 \pm 0.02 \mathrm{v} \\
(-82.84)\end{array}$ & $\begin{array}{l}5.0 \pm 0.0 \mathrm{mno} \\
(-52.83)\end{array}$ & 33 \\
\hline & 10 & $\begin{array}{l}1.16 \pm 0.01 p q \\
(-72)\end{array}$ & $\begin{array}{l}0.92 \pm 0.03 \mathrm{vw} \\
(-85.91)\end{array}$ & $\begin{array}{l}3.80 \pm 0.19 q r \\
(-64.15)\end{array}$ & 26 \\
\hline \multirow[t]{4}{*}{$\begin{array}{l}\text { Delonix } \\
\text { regia }\end{array}$} & 2.5 & $\begin{array}{l}4.46 \pm 0.07 b c \\
(7.2)\end{array}$ & $\begin{array}{l}5.32 \pm 0.03 g \\
(-18.52)\end{array}$ & $\begin{array}{l}8.20 \pm 0.19 \mathrm{~cd} \\
(-22.64)\end{array}$ & 89 \\
\hline & 5.0 & $\begin{array}{l}3.75 \pm 0.04 \mathrm{e} \\
(-9.8)\end{array}$ & $\begin{array}{l}4.10 \pm 0.01 \mathrm{kl} \\
(-37.21)\end{array}$ & $\begin{array}{l}7.80 \pm 0.19 \mathrm{cde} \\
(-26.41)\end{array}$ & 76 \\
\hline & 7.5 & $\begin{array}{l}2.54 \pm 0.04 i \\
(-39)\end{array}$ & $\begin{array}{l}2.15 \pm 0.02 \mathrm{~s} \\
(-67.07)\end{array}$ & $\begin{array}{l}6.0 \pm 0.0 \mathrm{ijk} \\
(-43.39)\end{array}$ & 50 \\
\hline & 10 & $\begin{array}{l}1.17 \pm 0.02 p q \\
(-72)\end{array}$ & $\begin{array}{l}0.97 \pm 0.05 \mathrm{v} \\
(-85.14)\end{array}$ & $\begin{array}{l}4.60 \pm 0.23 o p \\
(-56.60)\end{array}$ & 29 \\
\hline
\end{tabular}




\begin{tabular}{|c|c|c|c|c|c|}
\hline \multirow[t]{4}{*}{$\begin{array}{l}\text { Ziziphus } \\
\text { jujube }\end{array}$} & 2.5 & $\begin{array}{l}4.37 \pm 0.06 \mathrm{c} \\
(5)\end{array}$ & $\begin{array}{l}5.10 \pm 0.01 d \\
(-21.89)\end{array}$ & $\begin{array}{l}8.60 \pm 0.23 b c \\
(-18.86)\end{array}$ & 88 \\
\hline & 5.0 & $\begin{array}{l}4.42 \pm 0.04 c \\
(6.2)\end{array}$ & $\begin{array}{l}6.09 \pm 0.01 d \\
(-6.73)\end{array}$ & $\begin{array}{l}7.80 \pm 0.23 \mathrm{de} \\
(-26.41)\end{array}$ & 91 \\
\hline & 7.5 & $\begin{array}{l}3.19 \pm 0.04 \mathrm{~g} \\
(-23)\end{array}$ & $\begin{array}{l}5.78 \pm 0.05 \mathrm{e} \\
(-11.48)\end{array}$ & $\begin{array}{l}7.0 \pm 0.0 \mathrm{fgh} \\
(-33.96)\end{array}$ & 77 \\
\hline & 10 & $\begin{array}{l}3.17 \pm 0.01 \mathrm{~g} \\
(-23)\end{array}$ & $\begin{array}{l}4.83 \pm 0.06 \mathrm{i} \\
(-26.03)\end{array}$ & $\begin{array}{l}5.80 \pm 0.23 \mathrm{jkl} \\
(-45.28)\end{array}$ & 68 \\
\hline \multirow[t]{4}{*}{$\begin{array}{l}\text { Ziziphus } \\
\text { mauritiana }\end{array}$} & 2.5 & $\begin{array}{l}3.19 \pm 0.03 g \\
(-23)\end{array}$ & $\begin{array}{l}7.16 \pm 0.02 \mathrm{a} \\
(-9.64)\end{array}$ & $\begin{array}{l}7.80 \pm 0.19 \mathrm{de} \\
(-26.41)\end{array}$ & 87 \\
\hline & 5.0 & $\begin{array}{l}3.35 \pm 0.03 f \\
(-19.5)\end{array}$ & $\begin{array}{l}6.21 \pm 0.0 \mathrm{~cd} \\
(-4.90)\end{array}$ & $\begin{array}{l}6.80 \pm 0.19 f g h \\
(-35.84)\end{array}$ & 80 \\
\hline & 7.5 & $\begin{array}{l}2.77 \pm 0.05 h \\
(-33.4)\end{array}$ & $\begin{array}{l}3.43 \pm 0.07 n \\
(-32.15)\end{array}$ & $\begin{array}{l}5.60 \pm 0.23 \mathrm{klm} \\
(-47.16)\end{array}$ & 57 \\
\hline & 10 & $\begin{array}{l}1.38 \pm 0.09 \mathrm{o} \\
(-67)\end{array}$ & $\begin{array}{l}1.84 \pm 0.06 \mathrm{t} \\
(-71.82)\end{array}$ & $\begin{array}{l}5.20 \pm 0.191 \mathrm{mno} \\
(-50.94)\end{array}$ & 37 \\
\hline
\end{tabular}

Data are presented as mean \pm SEM; mean values having different letters in a column are statistically different at $p<0.05$; Values in parenthesis show \% (+) increase or (-) decrease as compared with control.

\section{Discussion}

Allelopathic extract of donor plant species on seedling growth (root and shoot length and dry biomass) was variable depending upon the concentration and species (Table 2). Root length, shoot length and seedling dry weight are three important growth indictors to assess phytotoxic effect of allelochemicals and herbicides (Kamble, 2006, Mushtaq et al., 2010a). Sometimes it is difficult to compare large data and decide most phytotoxic allelopathic plants when multiple plant species and extract concentrations are used as the impact on the three growth parameters may be different (Akhtar et al., 2010, Piyatida and Kato-Noguchi, 2010). In the present study; therefore, seedling persistence index was calculated, which accounts for root and shoot length as well as seedling dry weight to set a criterion for easy and simple comparison of many species and concentrations. Based on seedling persistence index, four plant species Moringa oleifera, Mangifera indica, Albizia procera and Delonix regia resulted in highest bioherbicidal potential (seedling persistence index $<30 \%$ of control) at $10 \%$ leaf extract against target plant species, Lepidium sativum. The phytotoxic effect was less at lower leaf extract concentrations. Few plant species showed a growth promontory effect at lowest leaf extract concentration $(2.5 \%)$. This is the characteristic of allelochemicals from plant extract that they inhibit growth of other plants at higher concentrations and promote at lower concentrations (Akhtar et al., 2010, Haq et al., 2010).

The bioherbicidal effect of plant species might be due to presence of allelochemicals in the leaf extract, which is also supported by higher total phenolic contents in most phytotoxic plant species except Albizia procera (Figure 1). Phenolic compounds are the largest class of allelochemicals inhibiting growth of various crop and weed species (John, 2012). Previously, allelochemicals have been isolated and identified from leaves of Moringa oleifera, Mangifera indica and Delonix regia (Chou and Leu, 1992; Bennett et al., 2003; Saleem et al., 2013). The allelochemicals from these plant species have shown bioherbicidal potential against weed species. Albizia procera was among the most phytotoxic four plant species; however, total phenolic contents were very low in it as compared with other species. It is likely that Albizia procera might have allelochemicals other than phenolic compounds (Koodkaew et al., 2012).

Though allelopathic leaf extract of the plant species in the present study significantly delayed germination of test species as indicated by enhanced time to start germination and mean germination time, the final germination was only inhibited by two species (Table 1). It has been reported that allelochemicals did not cause significant reduction in germination of target species, rather they inhibit early seedling growth (Mushtaq et al., 2010a). The reduction of early seedling growth is very critical regarding control of weeds because critical period for weed-crop competition starts with germination of crop from very $1^{\text {st }}$ week and lasts until 4-6 weeks after sowing (Kasasian and Seeyave, 1969). Furthermore, most herbicides are applied as early postemergence or postemergence to control weeds in field crops (Carey and Kells, 1995; Mushtaq et al., 2010b).

There is a dire need to explore new phytotoxins with diverse mode of actions for managing herbicide resistant weeds in agroecosystems worldwide (Dayan and Duke, 2014). The leaf extract of tested plant species may be exploited as bioherbicide or to develop eco-friendly future herbicides. However, future studies might be conducted to investigate allelopathic impact of these plant species against 
weeds under field conditions before general recommendation. In conclusion, among eight species investigated, four (Moringa oleifera, Mangifera indica, Albizia procera and Delonix regia) were most phytotoxic and have potential to be exploited as eco-friendly bioherbicides.

\section{References}

Akhtar, H., A. Kausar, M. Akram, Z.A. Cheema, I. Ali and M.N. Mushtaq. 2010. Effects of Dalbergia sissoo Roxb. leaf extract on some associated crop species of agroforestry. Allelopathy Journal 25: 221-226.

Alshahrani, H.S., R.R. Hicks, V. Sven and G.E. Siedel. 2009. Effects of leaf extracts of Zizyphus spina-christi and Prosopis juliflora on each other's seedlings roots. Allelopathy Journal 23: 111-118.

Bennett, R.N., F.A. Mellon, N. Foidl, J.H. Pratt, M.S. Dupont, L. Perkins and P.A. Kroon. 2003. Profiling glucosinolates and phenolics in vegetative and reproductive tissues of the multi-purpose trees Moringa oleifera L. (horseradish tree) and Moringa stenopetala L. Journal of Agricultural and Food Chemistry 51: 3546-3553.

Carey, J.B. and J.J. Kells. 1995. Timing of total postemergence herbicide applications to maximize weed control and corn (Zea mays) yield. Weed Technology 9: 356-361.

Cheng, F. and Z. Cheng. 2015. Research progress on the use of plant allelopathy in agriculture and the physiological and ecological mechanisms of allelopathy. Frontiers in Plant Science 6: 1020-1020.

Chou, C.-H. and L.-L. Leu. 1992. Allelopathic substances and interactions of Delonix regia (Boj) Raf. Journal of Chemical Ecology 18: 2285-2303.

Dayan, F.E. and S.O. Duke. 2014. Natural compounds as next generation herbicides. Plant Physiology 166: 1090-1105.

De, A., R. Bose, A. Kumar and S. Mozumdar. 2014. Worldwide pesticide use. p.5-6. In: Targeted Delivery of Pesticides Using Biodegradable Polymeric Nanoparticles. A. De, R. Bose, A. Kumar and S. Mozumdar (eds). Springer India, New Delhi.

Edwards, C.A. 2013. Environmental Pollution by Pesticides. Springer USA.

Ellis, R.H. and E.H. Roberts. 1981. The quantification of ageing and survival in orthodox seeds. Seed Science and Technology 9: 373-409.

Haq, R.A., M. Hussain, Z.A. Cheema, M.N. Mushtaq and M. Farooq. 2010. Mulberry leaf water extract inhibits bermudagrass and promotes wheat growth. Weed Biology and Management 10: 234-240.
Heap, I. 2018. The International Survey of Herbicide Resistant Weeds. Online. http://www.weedscience.org. Accessed 15/12/2018.

John, J. 2012. Role of phenolics in allelopathic interactions. Allelopathy Journal 29: 215-230.

Kamble, S.I. 2006. Effect of herbicide 2,4-D on seed germination and early seedling growth of Hibiscus cannabinus Linn. Biosciences Biotechnology Research Asia 31: 227-232.

Kasasian, L. and J. Seeyave. 1969. Critical periods for weed competition. PANS Pest Articles \& News Summaries 15: 208-212.

Koodkaew, I., S. Matsuyama, Y. Sunohara and H. Matsumoto. 2012. Hapalocyclamide: a novel phytotoxic hexapeptide of the Cyanobacterium hapalosiphon sp. Tetrahedron Letters 53: 977-979.

Macias, F.A., D. Castellano and J.M.G. Molinillo. 2000. Search for a standard phytotoxic bioassay for allelochemicals. selection of standard target species. Journal of Agricultural and Food Chemistry 48: 25122521.

Mushtaq, M., Z. Cheema and A. Khaliq. 2010a. Effects of mixture of allelopathic plant aqueous extracts on Trianthema portulacastrum L. weed. Allelopathy Journal 25: 205-212.

Mushtaq, M.N., Z.A. Cheema, A. Khaliq and M.R. Naveed. 2010b. A $75 \%$ reduction in herbicide use through integration with sorghum+sunflower extracts for weed management in wheat. Journal of the Science of Food and Agriculture 90:1897-1904.

Mushtaq, M.N., Y. Sunohara and H. Matsumoto. 2013. L-DOPA inhibited the root growth of lettuce by inducing reactive oxygen species generation. Weed Biology and Management 13: 129-134.

Perveen, S., M. Yousaf, M.N. Mushtaq, N. Sarwar, A. Khaliq and S. Hashim. 2019. Selective bioherbicidal potential of Delonix regia allelopathic leaf extract on germination and seedling growth of field bindweed and wheat. Applied Ecology and Environmental Research 17: 511-519.

Perveen, S., M. Yousaf, N. Sarwar, A.F .Zahoor, K. Saleem, M.N. Mushtaq and A. Anjum. 2015. Extraction, isolation and structure confirmaiton of cock's comb (Celosia argentia) leaves components for phytotoxic evaluation against Lepidium sativum. Oxidative Communicaitons 38: 141-149.

Phiri, C. and D.N. Mbewe. 2010. Influence of Moringa oleifera leaf extracts on germination and early seedling development of major cereals. International Journal of Agriculturei and Biology: 774-777. 
Piyatida, P. and H. Kato-Noguchi. 2010. Screening of allelopathic activity of eleven Thai medicinal plants on seedling growth of five test plant species. Asian Journal of Plant Sciences 9: 486-486.

Ranal, M.A. and D.G.D. Santana. 2006. How and why to measure the germination process? Brazilian Journal of Botany 29: 1-11.

Romano-Armada, N., M. Amoroso and V. Rajal. 2017. Effect of glyphosate application on soil quality and health under natural and zero tillage field condition. Soil and Environment 36(2): 141-154.

Saleem, K., S. Perveen, N. Sarwar, F. Latif, K.P. Akhtar and H.M.I. Arshad. 2013. Identification of phenolics in mango leaves extract and their allelopathic effect on canary grass and wheat. Pakistan Journal of Botany 45: 1527-1535.
Stokstad, E. 2013. Infographic: pesticide planet. Science 341: 730-731.

Tariq, K., A. Wahid, N. Maqbool, S. Nawaz and M. Islam. 2018. Possible role of molecular mass of medium supplemented phenolics in improving rice growth in puddled saline condition. Soil and Environment 37(2): 190-202.

Uddin, M., R. Ahmed, S. Mukul and M. Hossain. 2007. Inhibitory effects of Albizia lebbeck leaf extracts on germination and growth behavior of some popular agricultural crops.

Vaughn, S.F. and M.A. Berhow 1999. Allelochemicals isolated from tissues of the invasive weed garlic mustard (Alliaria petiolata). Journal of Chemical Ecology 25: 2495-2504. 